\title{
DESENVOLVIMENTO DE ESPÉCIES ARBÓREAS EM SISTEMAS AGROFLORESTAIS PARA RECUPERAÇÃO DE ÁREAS DEGRADADAS NA FLORESTA OMBRÓFILA DENSA, PARATY, RJ ${ }^{1}$
}

\author{
Márcia Cristina Soares de Souza² e Fatima Conceição Márquez Piña-Rodrigues ${ }^{3}$
}

\begin{abstract}
RESUMO - O objetivo deste trabalho foi avaliar o desenvolvimento de espécies arbóreas em diferentes modelos de sistemas agroflorestais empregados na recuperação de áreas degradadas na Floresta Ombrófila Densa, no Município de Paraty, RJ. Os sistemas agroflorestais implantados consistiram da combinação de espécies leguminosas e não leguminosas em plantio "solteiro" (uma planta/cova) e "casado" (duas plantas/cova). Aos quatro anos após o plantio, foram realizadas medições de circunferência na altura do peito (CAP), altura comercial (Hc) e altura total (Ht). Os resultados indicaram a eficiência do plantio "casado" nos sistemas agroflorestais, independente do uso de espécies leguminosas ou não leguminosas, indicando seu potencial de produção silvicultural para a recuperação de áreas degradadas.
\end{abstract}

Palavras-chave: Agrofloresta, Silvicultura, Mata Ciliar.

\section{EVALUTION OF FOREST SPECIES IN AGROFORESTRY SYSTEMS APPLIED TO RESTORATION OF DEGRADED AREAS AT OMBROPHYLOUS FOREST, PARATY, BRAZIL}

\begin{abstract}
The objective of this study was to evaluate the growth of forest species in different agroforestry systems applied to restoration of degraded areas in Paraty-RJ. The agroforestry systems were established with le guminous and non-leguminous species in "single" (one plant/hole) and "married" (two plants/hole) planting. To do so, circumference at breast height, commercial height and total height of the individuals were measured after four years of the planting. The results showed the efficiency of the "married" planting in the agroforestry systems, despite of using leguminous or non-leguminous species, indicating the silvicultural potential for restoration of degraded areas.
\end{abstract}

Keywords: Agroforestry, Riparian Forest, Silviculture.

\section{INTRODUÇÃO}

No final do século XVIII, praticamente toda a área de Floresta Ombrófila Densa no Município de Paraty, RJ, foi removida para o plantio de cana-de-açúcar. Posteriormente, parte dela foi naturalmente sucedida pela vegetação secundária que cobre, aproximadamente, $80 \%$ do território daquele município (PARATI, 2007). Segundo o levantamento de aptidão agrícola, apenas $10 \%$ da área do Município de Paraty é recomendada para o cultivo de lavoura ou pastagem, e o restante adequa-se ao manejo agroflorestal, reflorestamento e restauração das áreas de preservação permanente (BRASIL, 1983; PIÑA-RODRIGUES et al., 2006).

Nesse contexto, os Sistemas Agroflorestais (SAFs) apresentam-se como alternativas potenciais de uso do solo, uma vez que têm como objetivo recuperar as funções ambientais, aumentar a biodiversidade (SILVEIRA, 2003) e gerar produtividade agrícola, florestal

\footnotetext{
${ }^{1}$ Recebido em 23.02.2011 aceito para publicação em 17.01.2013

${ }^{2}$ Universidade Federal de São Carlos, Araras, SP.E-mail: <mcssouza06@ yahoo.com.br>.

${ }^{3}$ Universidade Federal de São Carlos, Sorocaba, Araras, SP.E-mail: <fpina@ufscar.br>.
} 
e pecuária (FRANCO et al., 2002). Ao mesmo tempo, há crescente preocupação governamental em estimular a recuperação de áreas de Reserva Legal (RL) e Áreas de Preservação Permanente (APP) com modelos de reflorestamento heterogêneos (RAMOS FILHO et al., 2006). Isso se aplica ao Estado de São Paulo na Instrução Normativa $n^{\circ} 47$, de 26 de novembro de 2003 (SEMA, 2003), na qual o plantio de SAFs pode ser viabilizado com esse objetivo. Contudo, a sua aplicação ainda carece de embasamento e diretrizes técnico-científicas sobre a eficiência do sistema em promover a restauração da produtividade e das funções ecológicas.

Entre os modelos agroflorestais que vêm sendo utilizados, o sistema regenerativo e análogo, também conhecido como SAFRA (VAZ, 2001), baseia-se na sucessão natural de espécies vegetais e animais, cuja dinâmica leva à maior complexidade do ambiente, de modo que o sistema produtivo seja semelhante à vegetação natural local em estrutura, composição e funcionalidade (SCHULTZ et al., 1994). Porém, apesar do potencial de restauração ecológica, o sucesso de um sistema agroflorestal também depende da escolha correta de seu componente arbóreo e de seu desenvolvimento. Por isso, devem ser também levados em conta os atributos silviculturais, como crescimento, sobrevivência, produção de lenho, frutos e sementes, os níveis de competição promovidos com os demais componentes, a adaptação às condições edafoclimáticas, a capacidade de rebrota, a baixa exigência nutricional e a baixa suscetibilidade às pragas e doenças (MACEDO, 2000).

As espécies arbóreas introduzidas nos SAFs têm a função de prestação de serviços ambientais, promovendo a cobertura dos solos, deposição de matéria orgânica via serrapilheira (MENDONÇA et al., 2001), redução de erosão e aumento da diversidade (GALZERANO, 2008). No entanto, também fazem parte do elemento produtivo pela geração de renda (ARATO et al., 2003), em especial por meio de produtos florestais, como madeira, frutos, sementes e óleos (BUDOWSKI, 1991). Nesse contexto, o consórcio com espécies arbóreas leguminosas favorece o desenvolvimento de outras plantas associadas, tanto pela produção de biomassa quanto pelos aspectos benéficos de sua capacidade de fixação biológica e interações com micorrizas (TILKI; FISHER, 1998). Seu uso em agroflorestas pode representar o aumento da produtividade geral do sistema e a melhoria da qualidade do solo, com reflexo no crescimento das plantas (MAGALHÃES, 2000).

Revista Árvore, Viçosa-MG, v.37, n.1, p.89-98, 2013
A falta de monitoramento contínuo de espécies agrícolas e florestais em sistemas agroflorestais é, contudo, um dos problemas que impedem o avanço do conhecimento do desempenho produtivo desses sistemas diversificados (GAMA, 2003). Diante disso, o plantio de espécies arbóreas e o acompanhamento de seu desenvolvimento por meio de medições periódicas da produtividade florestal são, portanto, importantes para orientar a escolha das espécies arbóreas e as formas de plantio (TONINI et al., 2006).

Dessa forma, o objetivo deste trabalho foi comparar o desenvolvimento de espécies arbóreas em diferentes modelos de sistemas agroflorestais, fornecendo informações que viabilizem seu uso na recuperação de áreas degradadas na Floresta Ombrófila Densa, no Município de Paraty, Rio de Janeiro.

\section{MATERIAL E MÉTODOS}

\section{1. Área de estudo}

O trabalho foi realizado em Áreas de Preservação Permanente (APPs) degradadas e recuperadas com sistemas agroflorestais, localizadas nas comunidades quilombolas, Campinho da Independência (23 $18^{\prime} 27^{\prime \prime}$

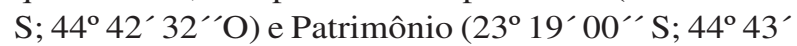
$23^{\prime \prime} \mathrm{O}$ ), situadas na Área de Proteção Ambiental (APA) do Cairuçu e no entorno do Parque Nacional da Serra da Bocaina, no distrito de Paraty-Mirim, no Município de Paraty, RJ. O clima da região é do tipo CWa, de acordo com a classificação de Köppen (1948). A precipitação anual média é de $2.384 \mathrm{~mm}$ e temperatura média anual de $27^{\circ} \mathrm{C}$ (MELLO, 2006). A cobertura vegetal original da área de estudo é do tipo Ombrófila Densa Submontana (IBGE, 1992).

\subsection{Descrição do sistema}

As unidades experimentais foram implantadas em março de 2003, baseadas no modelo de Sistemas Agroflorestais Regenerativos e Análogos (SAFRAs), com o objetivo de propor metodologias para Áreas de Preservação Permanente (APPs) com sistemas agroflorestais visando alternativas sustentáveis para a geração de renda em comunidades tradicionais (PIÑARODRIGUES et al., 2006). Cada unidade experimental (Campinho da Independência e Patrimônio) foi constituída de quatro parcelas de $20 \times 20 \mathrm{~m}$, correspondente aos tratamentos consorciando espécies leguminosas (T1) e não leguminosas (T3) com sistemas de plantio "solteiro" 
(uma muda por cova) e leguminosas (T2) e não leguminosas (T4) em sistema "casado" (plantio de duas mudas por cova) (Tabela 1). As mudas "casadas" no T2 foram guapuruvu (Schizolobium parahyba (Vell.) S.F. Blade) x ingá (Inga sp.), araribá (Centrolobium tomentosum Guill. ex Benth.) x embira-de-sapo (Lonchocarpus guillemineanus (Tul.) Malme), jatobá (Hymenaea courbaril L.) x banana (Musa sp.) e copaíba (Copaifera langsdorffii Desf.) x banana (Musa sp.) e no T4, cedro (Cedrela fissilis Vell.) x pau-viola (Citharexilum myriantum Cham.), canela (Nectandra lanceolata Ness) x urucum (Bixa ollerana L.), jequitibá (Cariniana legalis (Mart.) Kuntze) x banana (Musa sp.) e bicuíba (Virola bicuhyba (Schott ex Spreng.) Warb.) x banana (Musa sp.).

O espaçamento utilizado foi de 2,0 m entre linhas, alternando-se uma linha de plantio de pupunha (Bactris gasipaes Kunth) com outra linha de espécies arbóreas (solteiras) ou de consórcio de arbóreas e frutíferas (casadas). Dentro de cada linha, o espaçamento entre plantas de pupunha foi de $1,0 \mathrm{~m}$ e de $1,5 \mathrm{~m}$ entre as arbóreas e, ou, frutíferas. No sistema solteiro foram plantadas 719 mudas e no casado 842 , com um total de 462 mudas de pupunha por tratamento. No período de 2003 a 2006, foram plantadas espécies agrícolas, como feijão- preto (Phaseolus vulgaris L.), milho (Zea mays L.) e mandioca (Manihot esculenta L.) e de adubação verde, como feijão-de-porco (Canavalia ensiformis (L.) DC.), feijão-guandu (Cajanus cajans (L.) Millsp.) e crotalária (Crotalaria juncea L.), nas entrelinhas das espécies arbóreas.

\subsection{Análise de dados}

O crescimento das espécies arbóreas foi avaliado em outubro de 2007, quatro anos após a implantação das unidades experimentais, por meio de análise ao acaso de cinco indivíduos de cada espécie, obtendose medições das variáveis dendrométricas de Circunferência à Altura do Peito (CAP), Altura comercial (Hc) e Altura total (Ht). Os dados foram submetidos ao modelo geral linear (GLM) para realizar a análise de variância, envolvendo variáveis múltiplas e

Tabela 1 - Composição florística, sistemas de plantio e funções das espécies empregadas nos sistemas agroflorestais, Paraty, RJ. Table 1 - Floristic composition, planting systems and functions of the species used in the agroforestry systems, Paraty-RJ.

\begin{tabular}{|c|c|c|c|c|c|c|}
\hline \multirow[b]{2}{*}{ Tratamento } & \multirow[b]{2}{*}{$\begin{array}{c}\text { Sistema } \\
\text { de } \\
\text { plantio }\end{array}$} & \multirow[b]{2}{*}{$\begin{array}{l}\text { Espécie } \\
\text { arbórea }\end{array}$} & \multicolumn{4}{|c|}{ Espécies } \\
\hline & & & $\begin{array}{c}\text { Valor } \\
\text { econômico }\end{array}$ & $\begin{array}{l}\text { Frutíferas de ciclos } \\
\text { curto e longo }\end{array}$ & $\begin{array}{l}\text { Renovadoras de } \\
\text { fertilidade }\end{array}$ & $\begin{array}{l}\text { Uso potencial de sementes, } \\
\text { óleos e madeira de lei }\end{array}$ \\
\hline \multirow[t]{2}{*}{$\mathrm{T} 1$} & \multirow[t]{2}{*}{ Solteiro } & \multirow{3}{*}{ Leguminosa } & & Musa sp. (banana) & Inga sp. (ingá) & $\begin{array}{l}\text { Schizolobium parahyba (Vell.) } \\
\text { S.F. Blade (guapuruvu) }\end{array}$ \\
\hline & & & & $\begin{array}{l}\text { Carica papaya } \\
\text { (mamão) }\end{array}$ & $\begin{array}{l}\text { Lonchocarpus } \\
\text { guillemineanus }\end{array}$ & $\begin{array}{l}\text { Centrolobium tomentosum } \\
\text { Guill. ex Benth. (araribá) }\end{array}$ \\
\hline $\mathrm{T} 2$ & Casado & & Bactris & $\begin{array}{l}\text { Pouteria caimito } \\
\text { (Ruiz e Pav.) Radlk } \\
\text { (abiu) }\end{array}$ & $\begin{array}{l}\text { (Tul.) Malme } \\
\text { (embira-de-sapo) }\end{array}$ & $\begin{array}{c}\text { Hymenaea courbaril L. (jatobá) } \\
\text { Copaifera langsdorffii Desf. } \\
\text { (copaíba) }\end{array}$ \\
\hline \multirow{2}{*}{$\mathrm{T} 3$} & \multirow{2}{*}{ Solteiro } & \multirow{3}{*}{$\begin{array}{c}\text { Não } \\
\text { leguminosa }\end{array}$} & $\begin{array}{l}\text { gasipaes } \\
\text { Kunth } \\
\text { (pupunha) }\end{array}$ & $\begin{array}{c}\text { Artocarpus } \\
\text { heterophyllus Lam. } \\
\text { (jaca) }\end{array}$ & & Cedrela fissilis Vell. (cedro) \\
\hline & & & & $\begin{array}{l}\text { Anona muricata } \mathrm{L} \text {. } \\
\quad \text { (graviola) }\end{array}$ & $\begin{array}{l}\text { Bixa ollerana } \mathrm{L} . \\
\quad \text { (urucum) }\end{array}$ & $\begin{array}{l}\text { Nectrandra lanceolata Ness } \\
\text { (canela) }\end{array}$ \\
\hline $\mathrm{T} 4$ & Casado & & & $\begin{array}{l}\text { Averrhoa } \\
\text { carambola } \mathrm{L} . \\
\text { (carambola) }\end{array}$ & $\begin{array}{l}\text { Citharexilum } \\
\text { myriantum Cham. } \\
\text { (pau-viola) }\end{array}$ & $\begin{array}{l}\text { Cariniana legalis (Mart.) } \\
\quad \text { Kuntze (jequitibá) } \\
\text { Virola bicuhyba (Schott ex } \\
\text { Spreng.) Warb. (bicuíba) }\end{array}$ \\
\hline
\end{tabular}

Obs.: Solteiro - uma muda/cova; e Casado - duas mudas/cova; "Single" - one plant/cove; "Married" - two plants/cove. 
dependentes. Foi aplicado o teste de Tukey a 5\% de probabilidade, para a comparação das médias; e o método de Levene's, para a avaliação da homogeneidade da variância dos dados analisados com uso do programa SPSS 13.0 (McCUNE; GRACE, 2002).

A partir das variáveis circunferência (CAP), altura comercial $(\mathrm{Hc})$ e altura total $(\mathrm{Ht})$ foram calculados o incremento médio anual para altura ( IMA $\left._{\mathrm{HH}}\right)$ e diâmetro (IMA $_{\text {DAP }}$ ), adaptado de Cunha et al. (2002). Os dados de circunferência foram convertidos para diâmetro (DAP), calculando-se a relação Ht/DAP. Para as características de crescimento de cada espécie foram obtidas as médias de plantas por parcela. As análises em relação ao crescimento das espécies se concentraram na variável altura, porque esta reflete a diferenciação entre elas nos diferentes estádios sucessionais em florestas tropicais (BAKER et al., 2003). A homogeneidade de altura e de diâmetro foi analisada, calculando-se o coeficiente de variação para essas variáveis (ZAR, 1999).

\section{RESULTADOS}

O crescimento médio em Circunferência à Altura do Peito (CAP), Altura comercial (Hc) e Altura total (Ht) das espécies arbóreas aos 4 anos de idade foi significativamente diferente entre as unidades experimentais $\left(\mathrm{F}_{\mathrm{CAP}}=26,18 ; \mathrm{F}_{\mathrm{Hc}}=8,91 ; \mathrm{F}_{\mathrm{Ht}}=30,94 ; \mathrm{p}\right.$ $>0,05)$. As espécies arbóreas do Patrimônio apresentaram maiores taxas de desenvolvimento (CAP, Hc e Ht) em relação às do Campinho da Independência (Tabela 2).

Embora as condições dentro de cada unidade experimental tenham sido homogêneas, houve alta variabilidade no crescimento das plantas. Entre as espécies empregadas nos sistemas agroflorestais, pupunha, araribá e guapuruvu apresentaram maiores crescimentos em altura comercial e total, enquanto bicuíba foi aquela com as menores taxas (Tabela 2). O crescimento médio em circunferência das espécies apresentou comportamento semelhante ao da altura, porém o menor diâmetro foi observado em copaíba (Tabela 2).

Nas espécies arbóreas nativas empregadas nas unidades experimentais de sistemas agroflorestais, tanto nas leguminosas quanto nas não leguminosas o IMA em altura total foi alto para pupunha, araribá e guapuruvu, seguidos pelo ingá, canela e cedro. Entre as arbóreas frutíferas, jaca, abiu e graviola apresentaram os maiores incrementos em altura e diâmetro (Tabela 3).
Em relação aos modelos de sistemas agroflorestais testados, houve diferença significativa em relação às espécies leguminosas e não leguminosas para todas as variáveis analisadas $\left(\mathrm{F}_{\mathrm{CAP}}=6,01 ; \mathrm{F}_{\mathrm{Hc}}=13,26\right.$; $\left.\mathrm{F}_{\mathrm{Ht}}=6,01 ; \mathrm{p}<0,01\right)$, com maior desenvolvimento médio para as leguminosas (T1 e T2), principalmente no plantio "casado" (T2) (Figura 1). Entre as não leguminosas (T3 e T4), o plantio "casado" (T4) foi o que apresentou maiores taxas de crescimento, tendo sido significativamente diferente para CAP e altura total.

Ao analisar o comportamento das espécies leguminosas, guapuruvu e araribá apresentaram maiores crescimentos no sistema de plantio "casado" quando comparado com o "solteiro", enquanto ingá foi indiferente ao sistema de plantio (Figura 1). Entre as espécies não leguminosas, cedro e jequitibá apresentaram maiores alturas no plantio "casado" e urucum e canela no plantio "solteiro".

\section{DISCUSSÃO}

Os altos valores de coeficiente de variação obtidos nas unidades experimentais mostraram a heterogeneidade de ocupação do espaço pelas espécies presentes (Tabela 2), indicando a existência de processo de estratificação vertical (altura) e horizontal (diâmetro). Essa heterogeneidade, com coeficientes de variação maiores do que $60 \%$, foi mais acentuada na área do Patrimônio. Da mesma forma, Felfili (1995), em estudo conduzido em áreas de floresta natural, também observou crescimento variável, com valores de coeficiente de variação superiores a $60 \%$. Segundo essa autora, esse comportamento pode ser atribuído às condições de microssítios, a fatores genéticos e, mesmo, ao estabelecimento da competição entre plantas.

No caso de um sistema agroflorestal, em que todas as espécies foram plantadas simultaneamente, a estratificação pode representar uma aproximação com o sistema natural, uma vez que a heterogeneidade ambiental é uma característica desejável quando esses sistemas são aplicados para a recuperação de áreas degradadas. A estratificação favorece a diferenciação de nichos ecológicos, suportando maior diversidade de plantas e animais nos distintos estratos (HUNTERJÚNIOR, 1990), sendo um importante indicador de sustentabilidade ambiental, pois pode influenciar a riqueza, a diversidade, o crescimento e a produção de biomassa da comunidade (SOUZA et al., 2003). A

Revista Árvore, Viçosa-MG, v.37, n.1, p.89-98, 2013 
Tabela 2 - Crescimento médio em circunferência na altura do peito (CAP), altura comercial (Hc) e altura total (Ht) das espécies empregadas nos sistemas agroflorestais e coeficiente de variação (\%) obtidos nas espécies plantadas nas unidades experimentais de sistemas agroflorestais, Campinho da Independência e Patrimônio, Paraty, RJ.

Table 2 - Average growth in circumference at breast height (CAP), commercial height (Hc) and total height (Ht) of the species used in the agroforestry systems and coefficient of variation (\%) achieved by the forest species in the experimental plots of agroforestry systems, "Campinho da Independência" and "Patrimônio", Paraty-RJ.

\begin{tabular}{|c|c|c|c|c|c|c|c|}
\hline Espécie & Nome popular & \multicolumn{2}{|c|}{$\mathrm{CAP}(\mathrm{cm})$} & \multicolumn{2}{|c|}{$\mathrm{Hc}(\mathrm{m})$} & \multicolumn{2}{|c|}{$\mathrm{Ht}(\mathrm{m})$} \\
\hline Bactris gasipaes Kunth & Pupunha & \multicolumn{2}{|c|}{$51,9 \pm 22,4$} & \multicolumn{2}{|c|}{$4,7 \pm 2,3$} & \multicolumn{2}{|c|}{$8,4 \pm 3,0$} \\
\hline Centrolobium tomentosum Guill. ex Benth. & Araribá & \multicolumn{2}{|c|}{$28,9 \pm 7,0$} & \multicolumn{2}{|c|}{$7,5 \pm 6,4$} & \multicolumn{2}{|c|}{$8,1 \pm 1,9$} \\
\hline Schizolobium parahyba (Vell.) S.F. Blade & Guapuruvu & \multicolumn{2}{|c|}{$32,2 \pm 16,7$} & \multicolumn{2}{|c|}{$7,7 \pm 3,9$} & \multicolumn{2}{|c|}{$7,8 \pm 3,8$} \\
\hline Inga $\mathrm{sp}$. & Ingá & \multicolumn{2}{|c|}{$23,1 \pm 16,8$} & \multicolumn{2}{|c|}{$2,7 \pm 1,7$} & \multicolumn{2}{|c|}{$5,9 \pm 2,2$} \\
\hline Artocarpus heterophyllus Lam. & Jaca & \multicolumn{2}{|c|}{$15,2 \pm 14,4$} & \multicolumn{2}{|c|}{$4,0 \pm 6,0$} & \multicolumn{2}{|c|}{$3,8 \pm 2,6$} \\
\hline Pouteria caimito (Ruiz e Pav.) Radlk & Abiu & \multicolumn{2}{|c|}{$7,2 \pm 4,2$} & \multicolumn{2}{|c|}{$2,9 \pm 3,7$} & \multicolumn{2}{|c|}{$3,8 \pm 1,7$} \\
\hline Cariniana legalis (Mart.) Kuntze & Jequitibá & \multicolumn{2}{|c|}{$7,9 \pm 5,1$} & \multicolumn{2}{|c|}{$3,1 \pm 1,8$} & \multicolumn{2}{|c|}{$3,7 \pm 1,6$} \\
\hline Hymenaea courbaril L. & Jatobá & \multicolumn{2}{|c|}{$6,8 \pm 2,7$} & \multicolumn{2}{|c|}{$1,4 \pm 0,9$} & \multicolumn{2}{|c|}{$3,7 \pm 1,1$} \\
\hline Bixa ollerana $\mathrm{L}$. & Urucum & \multicolumn{2}{|c|}{$13,0 \pm 8,6$} & \multicolumn{2}{|c|}{$1,5 \pm 1,1$} & \multicolumn{2}{|c|}{$3,6 \pm 1,8$} \\
\hline Nectrandra lanceolata Ness & Canela & \multicolumn{2}{|c|}{$12,0 \pm 15,2$} & \multicolumn{2}{|c|}{$2,9 \pm 2,9$} & \multicolumn{2}{|c|}{$3,4 \pm 3,3$} \\
\hline Cedrela fissilis Vell. & Cedro & \multicolumn{2}{|c|}{$11,7 \pm 14,2$} & \multicolumn{2}{|c|}{$2,5 \pm 2,3$} & $3,2=$ & $\pm 2,9$ \\
\hline Anona muricata $\mathrm{L}$. & Graviola & 7,2 & $\pm 5,5$ & 1,7 & $\pm 1,7$ & $2,9=$ & $\pm 1,9$ \\
\hline Averrhoa carambola L. & Carambola & 5,2 & $\pm 3,3$ & 1,1 & $\pm 0,9$ & $2,4=$ & $\pm 1,1$ \\
\hline Citharexilum myriantum Cham. & Pau-viola & 5,4 & $\pm 8,7$ & 0,9 & $\pm 1,8$ & $2,0=$ & $\pm 2,4$ \\
\hline Lonchocarpus guillemineanus (Tul.) Malme & Embira-de-sapo & 4,3 & $\pm 4,3$ & 0,9 & $\pm 0,6$ & $1,9=$ & $\pm 1,2$ \\
\hline Copaifera langsdorffii Desf. & Copaíba & 2,9 & $\pm 4,2$ & 1,3 & $\pm 1,9$ & $1,5=$ & $\pm 1,9$ \\
\hline Virola bicuhyba (Schott ex Spreng.) Warb. & Bicuíba & 3,7 & $\pm 5,3$ & 1,2 & $\pm 1,5$ & $1,3=$ & $\pm 1,5$ \\
\hline Local & & Média & $\mathrm{CV}(\%)$ & Média & $\mathrm{CV}(\%)$ & Média & $\mathrm{CV}(\%)$ \\
\hline Campinho da Independência & & $11,6 \mathrm{~b}$ & $124,9 \mathrm{a}$ & $2,19 \mathrm{~b}$ & $88,2 \mathrm{~b}$ & $3,25 b$ & $69,4 b$ \\
\hline Patrimônio & & $18,0 \mathrm{a}$ & $113,4 \mathrm{~b}$ & $3,47 \mathrm{a}$ & $131,4 \mathrm{a}$ & $4,80 \mathrm{a}$ & $75,0 \mathrm{a}$ \\
\hline
\end{tabular}

Obs.: Espécies ordenadas com base no crescimento médio em altura total (Ht). Médias seguidas da mesma letra, dentro de cada variável, não diferem entre si, significativamente a $5 \%$ de probabilidade; Species ordered according to the average growth in total height (Ht). Means of each variable followed by the same letter do not differ by the Tukey's test at $5 \%$ of probability.

constatação da heterogeneidade reflete que o modelo de SAFRA promoveu a estratificação vertical e horizontal no sistema com valores similares aos da floresta natural, já aos 4 anos de idade.

As espécies que se destacaram no sistema agroflorestal testado foram a pupunha, a qual foi inserida com função de produção agrícola; o guapuruvu; e o araribá, ambos com função produtiva (madeira) e ecológica (fixação biológica e produção de biomassa) (Tabela 2). Confirmando o rápido desenvolvimento de guapuruvu e araribá, Piña-Rodrigues e Reis (2000) observaram que, aos 4 anos de idade, essas leguminosas arbóreas apresentaram altura média de 5,3 e 6,4 m, respectivamente, em plantios heterogêneos, realizados para recuperação de área degradada na Reserva Biológica de Poço das Antas, RJ. Oliveira-Neto et al. (2002), ao compararem o comportamento de Schizolobium parahybum var amazonicum em sistemas agroflorestais e monocultivos aos 40 meses de idade, constataram que nos plantios consorciados a média de altura total foi de 14,8 m, enquanto o monocultivo apresentou $13,7 \mathrm{~m}$. Piña-Rodrigues et al. (2000), ao estudarem o desenvolvimento de $S$. parahyba var. amazonicum, também consideraram a espécie promissora em plantios mistos devido ao seu alto incremento em altura e diâmetro. Dessa forma, esses resultados corroboram a indicação do uso de espécies do gênero Schizolobium em sistemas agroflorestais devido ao seu rápido desenvolvimento.

Em relação ao desenvolvimento das espécies, enquanto o crescimento em diâmetro é mais influenciado pela competição (SWAINE, 1994), a altura reflete as diferenças no gradiente vertical de irradiação, gerando variações no incremento em altura de espécies arbóreas em florestas tropicais (BAKER et al., 2003). Essas diferenças nas taxas de incremento em altura resultaram do crescimento distinto entre as espécies de diferentes grupos ecológicos. Como esperado, nas unidades experimentais as espécies pioneiras, de rápido crescimento e mais exigentes em luz, apresentaram 
Tabela 3 - Relação da altura total e do diâmetro à altura do peito (Ht/DAP), incremento médio anual em altura total (IMA Ht) e incremento médio anual em diâmetro (IMA DAP) das espécies arbóreas empregadas nas unidades experimentais de sistemas agroflorestais, Campinho da Independência e Patrimônio, Paraty, RJ.

Table 3 -Relationship between total height and diameter at breast height (Ht/DAP), annual average increment in total height (IMA Ht) and annual average increment in diameter (IMA DAP) of the species used in the experimental plots of agroforestry systems at "Campinho da Independência" and "Patrimônio", Paraty, RJ.

\begin{tabular}{|c|c|c|c|c|c|c|}
\hline \multirow{3}{*}{ Espécie } & \multicolumn{2}{|c|}{$\mathrm{Ht}(\mathrm{m}) / \mathrm{DAP}(\mathrm{cm})$} & \multicolumn{2}{|c|}{ IMA Ht (m.ano $\left.{ }^{-1}\right)$} & \multicolumn{2}{|c|}{ IMA DAP (cm.ano $\left.{ }^{-1}\right)$} \\
\hline & \multicolumn{2}{|c|}{ Local } & \multicolumn{2}{|c|}{ Local } & \multicolumn{2}{|c|}{ Local } \\
\hline & Campinho & Patrimônio & Campinho & Patrimônio & Campinho & Patrimônio \\
\hline Pouteria caimito & 177,4 & 172,6 & 0,7 & 1,1 & 0,4 & 0,7 \\
\hline C. tomentosum & 82,5 & 98,4 & 1,7 & 2,4 & 2,1 & 2,5 \\
\hline Virola bicuhyba & 144,6 & 112,1 & 0,4 & 0,8 & 0,3 & 0,8 \\
\hline N. lanceolata & 238,5 & 117,3 & 0,5 & 1,5 & 0,2 & 1,8 \\
\hline A. carambola & 134,2 & 183,6 & 0,7 & 0,6 & 0,6 & 0,3 \\
\hline Cedrela fissilis & 120,6 & 113,3 & 1,0 & 1,4 & 0,9 & 1,7 \\
\hline C. langsdorffii & 195,3 & 175,1 & 0,5 & 1,1 & 0,3 & 0,7 \\
\hline L. guillemineanus & 161,7 & 143,3 & 0,5 & 0,6 & 0,3 & 0,4 \\
\hline Anona muricata & 150,4 & 124,7 & 0,8 & 1,0 & 0,6 & 0,9 \\
\hline S.parahyba & 90,0 & 76,6 & 1,2 & 2,7 & 1,6 & 3,5 \\
\hline Inga sp. & 103,0 & 123,7 & 1,2 & 1,7 & 1,6 & 2,1 \\
\hline A. heterophyllus & 94,4 & 106,5 & 1,1 & 1,1 & 1,2 & 1,5 \\
\hline H. courbaril & 156,6 & 211,3 & 0,8 & 1,0 & 0,6 & 0,5 \\
\hline Cariniana legalis & 173,5 & 168,5 & 0,9 & 0,9 & 0,6 & 0,6 \\
\hline C. myriantum & 210,0 & 148,7 & 0,3 & 0,8 & 0,2 & 0,7 \\
\hline Bactris gasipaes & 66,9 & 53,4 & 1,5 & 2,6 & 3,2 & 5,0 \\
\hline Bixa ollerana & 119,0 & 102,4 & 0,7 & 1,1 & 0,7 & 1,3 \\
\hline
\end{tabular}

maior incremento tanto em altura quanto em diâmetro do que as não pioneiras, consideradas de crescimento lento e tolerantes à sombra (Figura 2).

$\mathrm{Na}$ área do Patrimônio, contudo, as condições locais e, ou, as práticas de manejo mais intensivas realizadas na área (PIÑA-RODRIGUES et al., 2006) podem ter favorecido o desenvolvimento das espécies não pioneiras, em especial no tratamento T4, uma vez que o crescimento em altura e em diâmetro foi superior em relação às pioneiras. Análises de solos e nutrientes realizadas em 2006 revelaram que houve aumento do $\mathrm{pH}$ em todos os tratamentos, em especial no T4, situado na área do Patrimônio, onde os teores de matéria orgânica foram significativamente maiores em relação aos demais (PIÑA-RODRIGUES et al., 2006). Esse resultado pode ter influenciado o maior desenvolvimento das espécies não pioneiras como consequência da melhoria da qualidade do solo.

Em ambas as áreas estudadas, Campinho da Independência e Patrimônio, os incrementos diamétricos foram superiores aos observados em florestas ombrófilas montanas tropicais e subtropicais, os quais variaram de 0,10 - 0,23 $\mathrm{cm}^{2} \mathrm{ano}^{-1}$ a $0,78 \mathrm{~cm}^{\mathrm{ano}}{ }^{-1}$ (WILLIAMSLINERA, 1996). No entanto, nas áreas de estudo os valores médios de incremento em altura obtidos foram inferiores aos registrados por Tonini et al. (2006), que observaram incrementos de 3,5 $\mathrm{m}^{2} \mathrm{ano}^{-1}$ para a espécie leguminosa pioneira Acacia mangium após 48 meses. Porém, esses resultados foram similares aos obtidos no sistema SAFRA em Paraty para araribá e guapuruvu e inferior à pupunha nos diferentes modelos utilizados (Tabela 3). Desse modo, ao aplicar esses dados como indicadores, a produtividade obtida no sistema agroflorestal pode ser considerada como superior ao das florestas tropicais e subtropicais. Com isso, o sistema atende, neste quesito, à condição de gerar produtividade florestal, citada como uma das características desejáveis dos agroecossistemas (DANIEL et al., 1999).

Em termos silviculturais, o incremento médio pode permitir a estimativa da colheita da produção florestal. Entre as leguminosas arbóreas, tanto guapuruvu quanto 

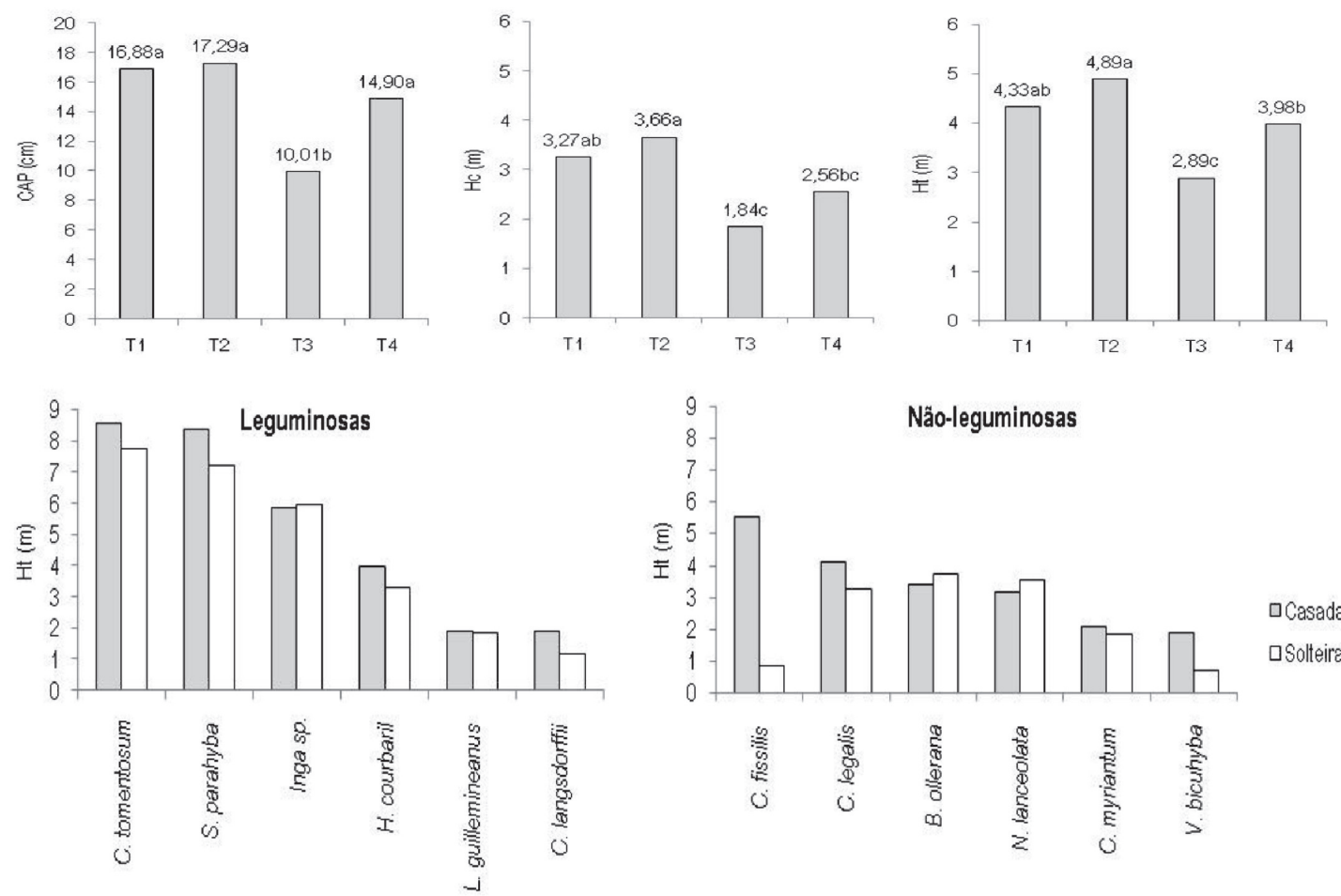

casada

口Solteira

Figura 1 - Crescimento médio em circunferência na altura do peito (CAP), altura comercial (Hc) e altura total (Ht) das espécies leguminosas e não leguminosas empregadas nos métodos "casado" (duas plantas/cova) e "solteiro" (uma planta/cova), Paraty, RJ. Médias seguidas de mesma letra indicam que os tratamentos não diferem estatisticamente entre si, pelo teste de Tukey a 5\% de probabilidade. T1 - leguminosas com plantio "solteiro"; T2 - leguminosas com plantio "casado"; T3 - não leguminosas com plantio "solteiro"; e T4 - não leguminosas com plantio "casado".

Figure 1 -Average growth in circumference at breath height (CAP), commercial height (Hc) and total height (Ht) of the leguminous and non-leguminous species employed in the "married" (two plants/hole) and "single" (one/hole) planting methods in the agroforestry systems, Paraty-RJ. Means followed by the same letter show that the treatments do not differ statistically from each other by the Tukey's test at $5 \%$ of probability. T1 - Leguminous with "single" planting; T2 - Leguminous with "married" planting; T3 - Non leguminous with "single" planting; and T4 - Non leguminous with "married" planting.

araribá, cujo diâmetro comercial médio para corte é de $20-25 \mathrm{~cm}$, teoricamente poderiam atingir esse valor entre 7 e 9 anos, caso o IMA de diâmetro se mantivesse constante. Com base nesses dados, essas espécies seriam altamente promissoras para o plantio em sistemas agroflorestais. Nas demais plantas inseridas no sistema agroflorestal, pupunha foi a que apresentou maior crescimento em altura e diâmetro, confirmando que, até os 4 anos, pode ser empregada em sistemas agroflorestais como uma espécie de produção em curto prazo, sem perda de produtividade em crescimento. Seu potencial de uso é ressaltado ainda por sua característica de precocidade na produção de palmito (TONET et al., 1999).
Os resultados deste estudo ressaltam a complexidade dos sistemas agroflorestais e apontam para a eficiência do plantio "casado", independente do uso de espécies leguminosas ou não leguminosas. Embora a presença de duas mudas por cova pudesse gerar maior competição entre elas, o plantio "casado" em sistemas agroflorestais não comprometeu o desenvolvimento dos indivíduos, confirmando a afirmação de Osterroht (2002), segundo o qual as árvores tendem a crescer melhor ao terem "intensa companhia". Com base nesses resultados, pode-se sugerir que as diferenças observadas entre os plantios "casados" e "solteiros" podem advir também das características ecológicas das espécies e do tipo de consórcio realizado, além de sua interação com o próprio sistema de plantio, o modelo SAFRA.

Revista Árvore, Viçosa-MG, v.37, n.1, p.89-98, 2013 

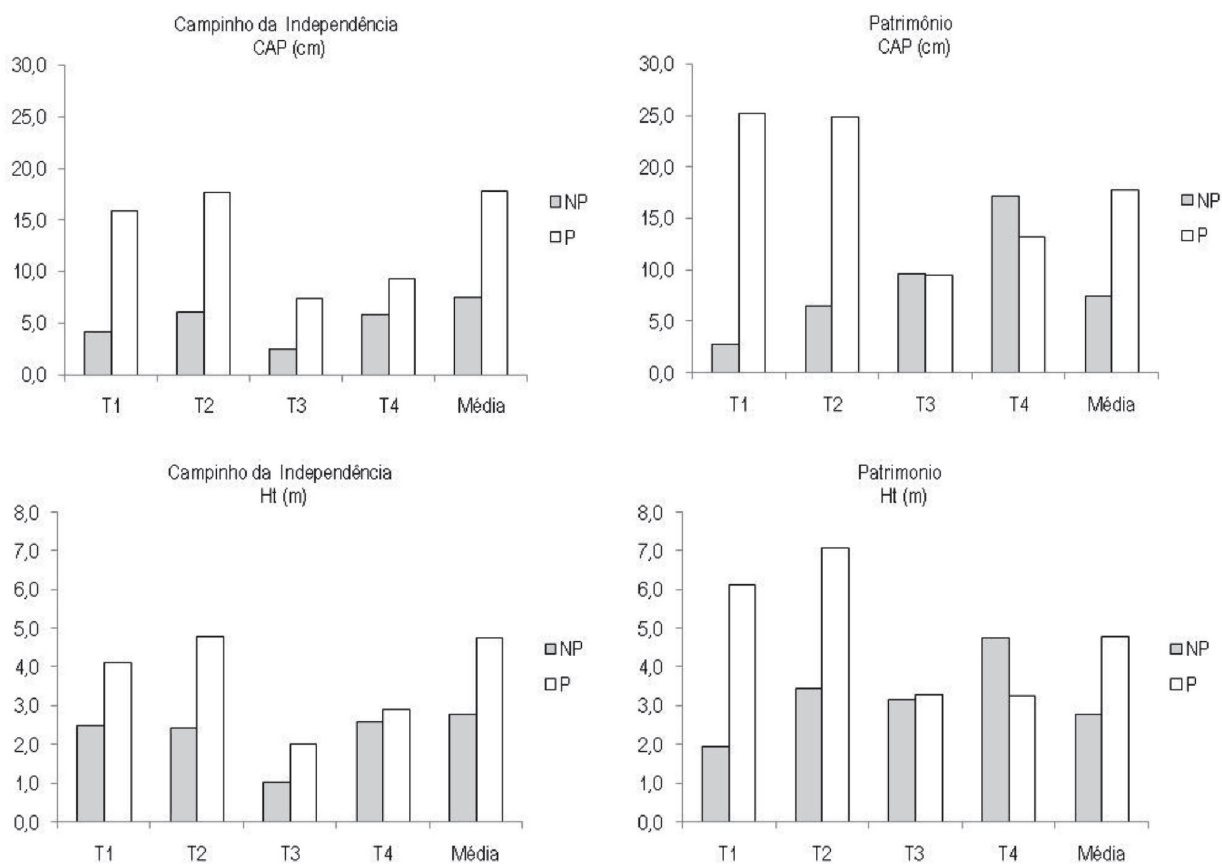

Figura 2 - Crescimento médio em circunferência na altura do peito (CAP) e altura total (Ht) dos grupos ecológicos nas unidades experimentais de sistemas agroflorestais, Campinho da Independência e Patrimônio, Paraty, RJ. Médias seguidas de mesma letra indicam que os tratamentos não diferem estatisticamente entre si, pelo teste de Tukey $(\mathrm{p}=0,05)$. T1 - leguminosas com plantio "solteiro"; T2 - leguminosas com plantio "casado"; T3 - não leguminosas com plantio "solteiro"; T4 - não leguminosas com plantio "casado"; P - pioneiras; e NP - não pioneiras.

Figure 2-Average growth in circumference at breast height (CAP) and total height (Ht) of ecological groups in the experimental plots of agroforestry systems, "Campinho da Independência" and "Patrimônio", Paraty-RJ. Means followed by the the same letter show that the treatments do not differ statistically from each other by the Tukey's test at 5\% of probability. T1 - Leguminous with "single" planting; T2 - Leguminous with "married" planting; T3 - Non leguminous with "single" planting; T4 - Non leguminous with "married" planting; and P - pioneer; NP - non-pioneer.

\section{CONCLUSÃO}

As práticas de manejo dos sistemas agroflorestais das unidades experimentais podem ter promovido o desenvolvimento diferenciado das espécies.

As espécies pioneiras, guapuruvu e araribá foram as que apresentaram maior desenvolvimento em altura e diâmetro, enquanto entre as não pioneiras se destacaram o cedro e jequitibá.

Os modelos de sistemas agroflorestais combinados com leguminosas foram os que apresentaram maior desenvolvimento das espécies arbóreas.

O modelo SAFRA empregado com o plantio "casado", independente do uso de espécies leguminosas ou não leguminosas, apresenta potencial silvicultural para ser indicado como sistema agroflorestal produtivo.
O uso do modelo SAFRA em áreas de preservação permanente promoveu o estabelecimento de estratificação horizontal e vertical semelhante a florestas naturais, sendo esta uma das características ecológicas que indicam o estabelecimento de processos desejáveis para a recuperação de áreas degradadas.

\section{AGRADECIMENTOS}

Ao Ministério do Meio Ambiente, por intermédio do Projeto PDA Mata Atlântica em Paraty, RJ, pelo apoio financeiro; ao IDACO, coordenador do Projeto; aos proprietários das unidades experimentais $\mathrm{Sr}$. Domingos Martins e Sr. Eraldo Alves Filho, por permitirem a realização desta pesquisa; às comunidades quilombolas Campinho da Independência e Patrimônio, pela acolhida e atenção dada; e à Engenheira Florestal Iliana Salgado 
e aos monitores do Projeto PDA - Paraty, pela ajuda na coleta dos dados de campo.

\section{REFERÊNCIAS}

ARATO, H. D.; MARTINS, S. V.; FERRARI, S. H. S. Produção e decomposição de serrapilheira em um sistema agroflorestal implantado para recuperação de área degradada em Viçosa, MG. Revista Árvore, v.27, n.5, p.715-721, 2003.

BAKER, T. R.; SWAINE, M. D.; BURSLEM, D. F. R. P. Variation in tropical forest growth rates: combined effects of functional group composition and resource avalability. Perspectives in Plant Ecology, Evolution and Systematics, v.6, n.1/2, p.21-36, 2003.

BRASIL. Ministério das Minas e Energia. Departamento Nacional da Produção Mineral. Projeto RADAM. Levantamento de recursos naturais: geologia, geomorfologia, solos, vegetação, uso potencial da terra. Rio de Janeiro: [s.n.], 1983. v.32.775p.

BUDOWSKI, G. Aplicabilidad de los sistemas agroflorestais. In: SEMINÁRIO SOBRE PLANEJAMENTO DE PROJETOS AUTOSUSTENTÁVEIS DE LENHA PARA AMÉRICA LATINA E CARIBE, 1991, Turrialba. Anais... Turrialba: FAO, 1991. v.1.p.161-167.

CUNHA, U. S.; MACHADO, S. A.; FIGUEIREDO FILHO, A. Uso de análise exploratória de dados e de regressão robusta na avaliação de crescimento de espécies comerciais de terra-firme da Amazônia. Revista Árvore, v.26, n.4, p.391-402, 2002.

DANIEL, O.; COUTO, L.; VITORINO, A. C. T. Sistemas agroflorestais como alternativas sustentáveis à recuperação de pastagens degradadas. In: SIMPÓSIO SUSTENTABILIDADE DA PECUÁRIA DE LEITE NO BRASIL, 1., 1999, Goiânia. Anais... Juiz de Fora: Embrapa-CNPGL, 1999. p.151-170.

FRANCO, F. S. et al. Quantificação de erosão em sistemas agroflorestais e convencionais na Zona da Mata de Minas Gerais. Revista Árvore, v.26, n.6, p.751-760, 2002.

FELFILI, J. M. Diversity, structure, and dynamics of a gallery forest in central Brazil. Vegetatio, v.117, p.1-15, 1995.
GALZERANO, L. Eucalipto em sistemas agrossilvipastoris. REDVET. Revista Electrónica de Veterinaria, v.9, n.3, p.1-6, 2008.

GAMA, M. M. B. Análise técnica e econômica de sistemas agroflorestais em Machadinho D'oeste, Rondônia. 2003. 112 f. Tese (Doutorado em Ciência Florestal) Universidade Federal de Viçosa, Viçosa, MG, 2003.

\section{INSTITUTO BRASILEIRO DE GEOGRAFIA E ESTATÍSTICA - IBGE. Manual técnico da vegetação brasileira. Rio de Janeiro: 1992. 92p. (Manuais Técnicos em Geociências, 1).}

KÖPPEN, W. Climatologia: con un estudio de los climas de la tierra. México: Fondo de Cultura Econômica, 1948. 479p.

HUNTER-JÚNIOR, M. L. Wildlife forests, and forestry: principles of managing forests for biological diversity. New Jersey: Prentice-Hall, 1990. 370p.

MACEDO, R. L. G. Fundamentos básicos para a implantação e manejo de sistemas agroflorestais. In: MACEDO, R. L. G. Princípios básicos para o manejo sustentável de sistemas agroflorestais. Lavras: UFLA/Faepe, 2000.

MAGALHÃES, J. A. Avaliação de leguminosas arbóreas e arbustivas de múltiplo propósito em Rondônia. In: CONGRESSO BRASILEIRO DE SISTEMAS AGROFLORESTAIS, 3., 2000, Manaus. Anais... Manaus: 2000. v.1. p.42-47.

McCUNE, B.; GRACE, J. B. Analysis of ecological communities. Oregon: $\mathrm{MjM}$ Software Design, Gleneden Beach, 2002.

MELLO, D. Paraty estudante. Guaratinguetá: Frei Galvão, 2006. 75p.

MENDONÇA, E. S.; LEITE, L. F. C.; FERREIRA NETO, P. S. Cultivo de café em sistema agroflorestal: uma opção para recuperação de solos degradados. Revista Árvore, v.25, n.3, p.375-383, 2001.

OLIVEIRA-NETO, S. N. et al. Comportamento de paricá (Schizolobium amazonicum Ducke) em sistema agroflorestal com pimenta-do-reino (Piper nigrum L.) na Região Amazônica. In: CONGRESSO BRASILEIRO DE SISTEMAS AGROFLORESTAIS, 4., 2002, Ilhéus. Anais... Disponível em: <http:// www.sbsaf.org.br/anais/2002/trabalhos/1025.pdf>

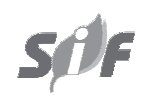

Revista Árvore, Viçosa-MG, v.37, n.1, p.89-98, 2013 
von OSTERROHT, M. Implantação de agroflorestas. Agroecologia Hoje, n.15, jul./ago., 2002.

PARATI (Município). História da cidade. Disponível em:<http://www.pmparaty.rj.gov.br>. Acesso em: 28 mar. 2007.

PIÑA-RODRIGUES, F. C. M. et al. Desenvolvimento de sistemas alternativos para a recuperação de áreas degradadas e geração de renda em comunidades tradicionais do entorno de unidades de conservação da Mata Atlântica. Seropédica: FAPUR, PRODETABParaty, 2006. 263p.

PIÑA-RODRIGUES, F.C.M.; REIS, L.L. Avaliação e monitoramento do crescimento de espécies arbóreas em sistema adensado para a recuperação de áreas degradadas na reserva biológica de Poço das Antas (Silva Jardim, RJ). In: SIMPÓSIO NACIONAL DE RECUPERAÇÃO DE ÁREAS DEGRADADAS, 4., 2000, Blumenau. Anais... Blumenau: Sociedade Brasileira de Recuperação de Áreas Degradadas, Fundação Universidade Regional de Blumenau, 2000. CD-ROM.

PIÑA-RODRIQUES, F. C. M. et al. Comportamento de paricá (Schizolobium amazonicum) e virola (Virola surinamensis) em plantios puros e mistos na Amazônia. In: SIMPÓSIO INTERNACIONAL SOBRE ECOSSISTEMAS FLORESTAIS, 6., 2000, Porto Seguro. Anais... Porto Seguro: Biosfera, 2000. p.73-74.

RAMOS FILHO, L. O. et al. Experiência participativa de sistemas agroflorestais no assentamento "Sepé Tiaraju”, Ribeirão Preto, SP. In: CONGRESSO BRASILEIRO DE

AGROECOLOGIA, 1., 2006, Belo Horizonte.

Anais... Belo Horizonte: 2006.

SCHULTZ, B.; BECKER, B.; GÖTSCH, E. Indigenous knowledge in a "modern" sustainable agroflorestry system - a case study from eastern Brazil. Agroforestry Systems, v.25, n.1, p.5969, 1994.
SÃO PAULO. Secretaria de Meio Ambiente do Estado de São Paulo. Resolução SMA no 47 de 23 de novembro de 2003.

SILVEIRA, N. D. Indicadores de sustentabilidade ambiental em sistemas agroflorestais na Mata Atlântica. 2003. 75 f. Monografia (Graduação em Engenharia Florestal) - Universidade Federal Rural do Rio de Janeiro, Seropédica, 2003.

SOUZA, D. R. et al. Emprego de análise multivariada para estratificação vertical de florestas ineqüiâneas. Revista Árvore, v.27, n.1, p.59-63, 2003.

SWAINE, M. D. Long term studies of tropical forest dynamics. In: JOHNSTON, A. E. Longterm experiments in agricultural and ecologycal sciences. Wallington: CAB Insternational, 1994. p.305-302.

TILKI, F.; FISHER, R. F. Tropical leguminous species for acid soils: studies on plant form and growth in Costa Rica. Forest Ecology and Management, v.108, p.175-192, 1998.

TONINI, H. et al. Avaliação de espécies florestais em área de mata no estado de Roraima. Cerne, v.12, n.1, p.8-18, 2006.

TONET, R. M.; FERREIRA, L. G. S.; OTOBONI, J. L. M. A cultura da pupunha. Campinas: Coordenadoria de Assistência Técnica Integral (CATI), 1999. 44p. (Boletim Técnico, 237)

VAZ, P. Agroforestería en Brasil: una experiencia de regeneración análoga. Lima: LEISA Boletín de ILEIA, 2001. p.5-7.

WILLIAMS-LINERA, G. Crecimiento diamétrico de arboles caducifolios y perennifolios del bosque mesófilo de montaña en los alrededores de Xalapa. Madera y Bosques, v.2, n.2, p.53-65, 1996.

ZAR, J. H. Biostatistical analysis. Upper Saddle River: Prentice Hall. 1999. 